\title{
Editorial
}

\section{Celebrating our tenth anniversary and our community of practice}

\author{
Paula McGee PhD RN RNT MA BA Cert Ed \\ Editor, Diversity and Equality in Health and Care; Professor of Nursing, Faculty of Health, Birmingham City \\ University, Birmingham, UK \\ Mark RD Johnson MA PhD Dip HE (Warwick) \\ Editor, Diversity and Equality in Health and Care; Professor of Diversity in Health and Social Care, Mary \\ Seacole Research Centre, De Montfort University, Leicester, UK
}

2014 marks the tenth anniversary of the launch of this journal. We began with what seemed like the simple idea of creating a community of interdisciplinary scholarship that drew together different aspects of diversity at an international level. We saw this as important because although several other publications addressed specific aspects of diversity, none of them brought these together in a single publication or considered the relationships between them. We argued that, in maintaining the separation of discourses about, for example, gender, ethnicity, age and disability, the experiences common to all were overlooked. Although discrimination may be enacted and experienced in particular ways towards women or those with physical differences, the underlying attitudes and terminology reflect a devaluing of people because of what they are not. Women are devalued because they are not men, black people are devalued because they are not white, and wheelchair users are devalued because they cannot walk. The same language, and the same perverse jokes, persist: 'There was an $\mathrm{X}$, a $\mathrm{Y}$ and a (woman).'

The UK's Equality Act (2010) was based on the recognition that British legislation had not kept pace with the growing understanding of diversity. Up to that point in time, UK law dealt only with gender, race and disability alongside a broad notion of equal opportunities. The Equality Act (2010) itemised nine protected characteristics, namely age, being or becoming a transsexual person, having a disability, being married or in a civil partnership, being pregnant or having a child, being of a particular race, colour or nationality, being of a particular religion, being of a particular gender and being of a particular sexual orientation. It is illegal to discriminate against someone on these grounds in education, at work, as a customer, when using public services, when buying or selling property, or when a member or guest of a private club or association. In this, UK law reflects and to a certain extent goes beyond the generally accepted European collation of equality standards embodied in the updated Article 13 of the Amsterdam Treaty (europa.eu/legislation.../treaties/amsterdam_treaty/ a10000_en.htm), which amended the European Community treaty and extended the original European Convention on Human Rights and Fundamental Freedoms.

Diversity and Equality in Health and Care has developed to reflect changing understandings of diversity and the increasing recognition of intersectionality', a term and issue which we felt we understood and wished to address, intuitively, but did not at the time have a phrase for. We are delighted that the European Union's Fundamental Rights Authority has provided a handy glossary on multiple discrimination (http://fra.europa.eu/en/project/2011/ multiple-discrimination-healthcare) to help to explore this.

Over the 10 years since the launch of this journal we have changed our name twice, first from Diversity in Health and Social Care to Diversity in Health and Care, which was easier to write, and better reflected the mix of papers and correspondence we were receiving. More recently, we have incorporated the notion of equality in our title, as this became more clearly the goal rather than mere determination of, and opposition to, discrimination. We are proud and pleased to note that our authors and readers have welcomed this change, and that they have been submitting ever more sophisticated papers, bringing together both qualitative and quantitative perspectives, along with a growing number of studies on sexual orientation and disability. At the same time, we hope that we have retained our interest and focus on discrimination and inequality arising from diversity in ethnicity, language, religion and nationality or migrant origin. As editors, 
with our expanding and increasingly international editorial board, whose contribution we acknowledge here, we expect and intend to continue to evolve to reflect the changing needs and interests of our readers and the welfare systems that they research, manage or use. Plans for new initiatives will be announced here, and we welcome suggestions from our readers as well. In this context, we should perhaps mention our move into the 21st-century world of social media with a 'Twitter' feed, which you can use to share with us, or to keep yourselves updated on new issues and content (follow@DiversityJnl ).

Finally, we wish to thank Radcliffe Publishing and their new owners, Electric Word, for continuing to support this journal. One of the crucial ways in which we have worked with them to develop a journal that is responsive to the needs of researchers and readers is by introducing a Green/Gold Open Access route for publication, which may enable those whose funding permits it to obtain, with the usual quality criteria, open-access publishing of their outputs and an enhanced readership for their specialised diverse findings. This facility was launched in Volume 10, Issue 3, and has led to an immediate rise in readership and a surprising number of people accessing our items on diabetes care.

\section{In this issue}

Delivery of health and social care is dependent upon collaboration between members of different professions, each of which has professional purposes, standards and code of conduct that may or may not sit comfortably with those of the others. Perceptions of the patient/client and the professional role can be very different. Concepts such as confidentiality and collaboration may appear universal, but experience of multidisciplinary working quickly reveals that this is not the case. Collaboration, for example, is frequently misinterpreted as simply working together, but it involves far more than that. The basis of true collaboration is valuing, trusting and respecting not only one's own expertise but also that of everyone else in a coming together of equals to pursue an agreed goal (Hanson and Spross, 2009). Collaboration also requires recognition of one's own limitations and a willingness to concede that other team members may be better able to meet the need of a patient/client. Unfortunately, many aspects of health and social care services still depend on hierarchies in which particular individuals retain the right to veto others' decisions or recommendations, irrespective of whether these are in the best interests of the patient/client. In this context it is therefore refreshing to present, in this issue, papers that reflect attempts to move beyond this traditional way of working. These papers do not provide solutions that will suit every workplace, but they do demonstrate a willingness to address the needs of patients/ clients in new ways.

We begin with a guest editorial by Wanpen Pinyopasakul (2014) about the development of discharge planning in Thailand. The problems highlighted here are very similar to those found in many other countries, namely fragmentation of care, lack of continuity of care between hospital and community settings, systems that are dominated by medical specialties, the concentration of resources in large urban areas, and high costs. In Thailand, as elsewhere, there is a growing realisation that the current system is unsustainable and that people are better served by improvements in primary care. Primary healthcare is people centred rather than professionally or managerially focused: 'As societies modernise, and become more affluent and knowledgeable, what people consider to be desirable ways of living ... they also have other, rising expectations regarding health and health care. People care more about health as an integral part of how they and their families go about their everyday lives than is commonly thought. ... They want health care that deals with people as individuals with rights, and not as mere targets. ... People also have expectations about the way their society deals with health and health care. They aspire to greater health equity and solidarity and are increasingly intolerant of social exclusion (World Health Organization, 2008, p. 11). Primary care is therefore about inclusion, and the integration of disparate sectors in service provision, both local and needs driven. Formulating individualised plans represents a first step towards good-quality primary care, but considerable work lies ahead to establish the collaborative practices required to make it a reality.

Our three research papers provide two examples of how exclusion works in health and social care services. In the first paper, Peter Zeh and colleagues (2014) highlight the barriers to diabetes care experienced by members of minority ethnic groups. We have noted elsewhere that diabetes is a major public health problem worldwide; the complications of the disease are frequently severe and difficult to treat (McGee and Johnson, 2013). It is therefore vital that people with diabetes are identified as early as possible and receive appropriate treatment. It is also a matter of serious concern if people cannot access the help that is available. This paper demonstrates the multiple barriers to diabetes care that people may experience. Some of these arise from the language used to explain the disease. For example, where professionals and patients do not share the same language proficiency, the statement that 'Diabetes means you have too much sugar' may be understood as 'I should cut down the amount of sugar in my tea' and nothing more. Even 
when dietary advice has been understood, integrating it into daily life can create another seemingly insurmountable barrier (McGee and Johnson, 2008). The overcoming of barriers is therefore not a single event but a whole series of experiences that, for many people, never seem to change for the better and which eventually lead them to give up or seek alternatives away from mainstream healthcare.

Our second paper, by Eleni Hatzimitriadou and Maria Psoinos (2014), shows how healthcare professionals themselves may be excluded because they do not fit prevailing professional norms. This paper presents an account of the experiences of migrant healthcare professionals in the UK. The study participants were all highly qualified and experienced professionals in their home country, but found it extremely difficult to be recognised as such in the UK. Gaining acceptance of their qualifications was a struggle, particularly for doctors, whereas nurses tended to fare better. None of the professionals achieved the level of post that they had held in their home country. This paper coincided with the publication of the final report of the Mid Staffordshire NHS Foundation Trust Public Inquiry (Francis, 2013), which revealed neglect of patients in Mid Staffordshire Hospital, and the realisation that many more UK hospitals are desperately understaffed. As a result, the National Health Service (NHS) is now struggling to recruit staff, particularly nurses, and at least one-third of NHS trusts are trying to attract applicants from other countries (Lintern, 2013). Moreover, given the expanding diversity of the UK population, it would be helpful to recruit professionals who are familiar with the cultures and languages of migrants' countries of origin, and to begin with those whose abilities are currently underutilised.

Our third paper, by Bruce Kirkaldy and colleagues (2014), touches on health and exclusion, from a practitioner's perspective, in Germany. Like the UK, Germany is a destination country, particularly for migrants from Turkey, so barriers to health services may be present because of differences in language and culture. We have already seen that access to services is problematic for many adults, but finding help for children and adolescents is even more difficult, especially with regard to mental health problems. Communication between adults and between adults and children may present major problems. Perhaps the child is more fluent than the parent in the host country's language, and the parent feels excluded from the consultation, or maybe the child feels unable to communicate and that adults are talking about them. Such concerns prompted the authors to survey psychiatrists working with children and adolescents about working with migrant children and their families. This is our first paper about child and adolescent mental health, and one of the authors, Nisha Dogra, has also contributed a useful Continuing Professional Development feature (Dogra, 2014) for readers who are unfamiliar with this field.

Our two practice papers in this issue reflect attempts to ensure inclusion and people-centred care. Elizabeth Johnston Taylor and her colleagues (2014) present an account of their engagement with Māori people regarding end-of-life care in a New Zealand hospice. This paper highlights the distance between professionals and service users. The Māori understanding of the world differs in significant ways from that of the staff who are providing hospice care. Although Māori views of health were compatible with the values of hospice care, there was evidence of considerable distance over issues of language and the significance of singing and food in end-of-life care. Care that is truly person centred will incorporate and respect what is important to people. Professionals have to understand that the majority of individuals conceptualise their health, illnesses and death in terms of their relationships with others, and without much, if any, reference to what is happening to their bodies (Helman, 2007). This is not to say that professionals should abandon their knowledge and skills and just give people what they want. We do not advocate that professionals should abandon their responsibilities, but rather that they should, where possible, collaborate with patients and families in weaving together their two views of the world. On a different note, any reader who would like to learn some Māori words will find this article of particular interest.

Our other practice paper, by Roberto Ramos and colleagues (2014), shows what can be achieved when an organisation decides to demonstrate its commitment to inclusivity. This is an important element in bringing about change. Too often it is left to individual practitioners to do the best they can, sometimes with very limited resources and in the face of fierce opposition. In bringing about change, organisations have a responsibility to show that they mean business. This paper describes how one cancer centre in the USA created and implemented a comprehensive language service for use in the care of every patient who did not speak or read English sufficiently well to understand communications from professionals. The service includes interpreting by qualified interpreters, translation of important documents for use by patients, and an out-of-hours service. It has been integrated into all of the services provided by the centre, and patients who refuse an interpreter are told that he or she will be present anyway to assist the professionals. Systematic evaluation of the programme is continuing, and we look forward to hearing more about it at a future date.

Our Practitioner's Blog, by Mary Dawood and Sunil Sahota, continues this focus on interpreting by reflecting on the use of family members to interpret for patients who do not speak the same language as professionals 
in the emergency department. In Did You See?, Cheryle Moss, Pamela Wood and Katherine Nelson review a paper by Tula Brannelly and colleagues on some of the issues raised in our other practice paper about working with Māori people. Finally, we are pleased to welcome the first Knowledgeshare section edited by Nicky Hudson (nhudson@dmu.ac.uk). This section contains another first, namely news from the European Transcultural Nursing Association. Diversity and Equality in Health and Care is pleased to be affiliated with this organisation, and we hope that other associations will join with us in due course.

Twitter: If anyone wants to Tweet, we now have an @DiversityJnl identity which provides an alternative to contacting us via our email address (dhc@ radcliffepublishing.com). We intend to use the Twitter feed to ensure the widest possible coverage of the journal, and to highlight other issues or matters of concern to our readership. We welcome feedback and hope that this might make it easier for some of our readers to respond to what they have read in the journal, as well as to retweet and spread the word about us. Please also follow us!

\section{REFERENCES}

Dogra N (2014) Childhood mental illness. Diversity and Equality in Health and Care 11:00-00.

Francis R (2013) The Mid Staffordshire NHS Foundation Trust Public Inquiry. Final Report. London: The Stationery Office.

Hanson CM and Spross JA (2009) Collaboration. In: Hamric $\mathrm{AB}$, Spross JA and Hanson CM (eds) Advanced Practice Nursing: an integrative approach, 4 th edn. St Louis, MO: Saunders, Elsevier. pp. 283-314.
Hatzidimitriadou E and Psoinos M (2014) Cultural health capital and professional experiences of overseas doctors and nurses in the UK. Diversity and Equality in Health and Care 11:00-00.

Helman C (2007) Culture, Health and Illness, 5th edn. London: Hodder Arnold.

Johnston Taylor E, Simmonds S, Earp R et al (2014) Māori perspectives on hospice care. Diversity and Equality in Health and Care 11:00-00.

Kirkcaldy B, Furnham AF, Dogra N et al (2014) Attitudes towards treatment of migrant youth and their families among child and adolescent psychiatrists: current clinical practice and developmental perspectives. Diversity and Equality in Health and Care 11:00-00.

Lintern S (2013) Exclusive: nursing shortage drives hospitals overseas. www.nursingtimes.net/nursing-practice/clinical -zones/management/exclusive-nursing-shortage-driveshospitals-overseas/5064201.article

McGee P and Johnson M (2008) I never needed to know the word for diabetes till I took this job. Diversity in Health and Social Care 5:1-3.

McGee P and Johnson M (2013) Diabetes: a public health issue for the twenty-first century. Diversity and Equality in Health and Care 10:135-9.

Pinyopasakul W (2014) From past to present: discharge planning in Thailand. Diversity and Equality in Health and Care 11:00-00.

Ramos R, Davis JL, Antolino P et al (2014) Language and communication services: a cancer centre perspective. Diversity and Equality in Health and Care 11:00-00.

World Health Organization (2008) The World Health Report. Geneva: World Health Organization.

Zeh P, Harbinder K, Cannaby AM et al (2014) Cultural barriers impeding ethnic minority groups from accessing effective diabetes care services: a systematic review of observational studies. Diversity and Equality in Health and Care 11:00-00. 